\section{Fundus autofluorescence imaging: systematic review of test accuracy for the diagnosis and monitoring of retinal conditions}

GK Frampton¹, N Kalita1', L Payne ${ }^{2}$, JL Colquitt ${ }^{3}$, E Loveman ${ }^{3}$, SM Downes ${ }^{4}$ and AJ Lotery ${ }^{5}$

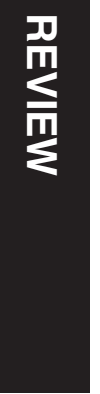

${ }^{1}$ Southampton Health Technology Assessments Centre (SHTAC), Faculty of Medicine, University of Southampton, Southampton, UK

recommendations to address this. Eye (2017) 31, 995-1007; doi:10.1038/eye.2017.19; published online 10 March 2017 conditions. Searches in November 2014 identified English language references. Sources included MEDLINE, EMBASE, the Cochrane Library, Web of Science, and MEDION databases; reference lists of retrieved studies; and internet pages of relevant organisations, meetings, and trial registries. For inclusion, studies had to report FAF imaging accuracy quantitatively. Studies were critically appraised using QUADAS risk of bias criteria. Two reviewers conducted all review steps. From 2240 unique references identified, eight primary research studies met the inclusion criteria. These investigated diagnostic accuracy of FAF imaging for choroidal neovascularisation (one study), reticular pseudodrusen (three studies), cystoid macular oedema (two studies), and diabetic macular oedema (two studies). Diagnostic sensitivity of FAF imaging ranged from 32 to $100 \%$ and specificity from 34 to $100 \%$. However, owing to methodological limitations, including high and/or unclear risks of bias, none of these studies provides conclusive evidence of the diagnostic accuracy of FAF imaging. Study heterogeneity precluded metaanalysis. In most studies, the patient spectrum was not reflective of those who would present in clinical practice and no studies adequately reported whether FAF images were interpreted consistently. No studies of monitoring accuracy were identified. An update in October 2016, based on MEDLINE and internet searches, identified four new studies but did not alter our conclusions. Robust quantitative evidence on the accuracy of FAF imaging and how FAF

\section{Introduction}

Fundus autofluorescence (FAF) is increasingly being used in ophthalmology for detecting or monitoring retinal pathology, since autofluorescent molecules, referred to as fluorophores, can provide potentially useful Extensive and focal accumulations of lipofuscin and other fluorophores usually indicate retinal pathology ${ }^{1,2}$ and result in patterns of increased (hyper) autofluoresence relative to those seen in a normal eye. In conditions involving atrophy of the retina, (eg, geographic atrophy) or where morphological changes, fluid accumulation, fibrosis, pigment changes, or haemorrhages obscure autofluorescence, decreased (hypo) autofluorescence may also signal retinal pathology. ${ }^{3,4}$ As a result, characteristic spatial distributions of hyper-autofluorescence and hypo-autofluorescence can be associated with a wide range of retinal conditions. ${ }^{1}$

The intensity of FAF is relatively weak (about two orders of magnitude lower than the background emission of a fluorescein angiogram at peak dye transit), ${ }^{3}$ meaning that specialist imaging techniques are required to enable FAF to be detected and mapped. Three main techniques have been used for FAF imaging, which are: fundus camera, fundus spectrophotometry, and confocal scanning laser diagnostic and prognostic information. ${ }^{1}$
${ }^{2}$ Department of Psychology, Faculty of Social, Human and Mathematical Sciences, University of Southampton, Southampton, UK

${ }^{3}$ Effective Evidence LLP, Waterlooville, UK

${ }^{4}$ Oxford Eye Hospital and John Radcliffe Hospital, Oxford, UK

${ }^{5}$ Faculty of Medicine, University of Southampton and Southampton University Hospital NHS Foundation Trust, Southampton, UK

Correspondence: GK Frampton, Southampton Health Technology Assessments Centre (SHTAC), Faculty of Medicine, University of Southampton, Epsilon House, Enterprise Road, Southampton Science Park, Southampton SO16 7NS, UK

Tel: +44 (0)23 8059 9299; Fax: +44 (0)23 80599299 E-mail: gkf1@soton.ac.uk

Received: 20 July 2016 Accepted in revised form: 2 December 2016 Published online: 10 March 2017 
ophthalmoscopy (cSLO). ${ }^{1,3}$ Compared to the other methods, cSLO is superior at minimising the detection of artefacts such as autofluorescence arising from structures anterior to the retina (eg, the cornea or lens). ${ }^{4}$ As such, cSLO is considered the most appropriate method currently available for measuring FAF. In cSLO, a focused low-power laser beam is swept across the fundus in a raster pattern to provide the excitatory light source for fluorophores. ${ }^{5}$ Excitation wavelengths include $488 \mathrm{~nm}$ (blue light) obtained with a solid-state laser and, developed more recently, $514 \mathrm{~nm}$ (green light) with an argon-ion laser. ${ }^{4}$ For near-infrared FAF, the excitation wavelength is $790 \mathrm{~nm}$. Unlike fundus spectrophotometry, cSLO can cover relatively large retinal areas $\left(\mathrm{eg}, 55^{\circ}\right.$ in Heidelberg retina angiograph (HRA)-based systems). A FAF image obtained from cSLO typically shows the spatial distribution of FAF intensity as pixels in greyscale values with dark pixels indicating low FAF intensity and bright pixels indicating high FAF intensity. ${ }^{3}$ The mean image of several FAF images is usually obtained to reduce background noise and enhance image contrast. To block reflected light but permit autofluorescence to pass, cSLO have barrier filters of 500, 525 and $800 \mathrm{~nm}$ for excitation wavelengths of 488, 514 and $790 \mathrm{~nm}$, respectively. Image contrast and brightness settings also differ among cSLO devices and these differences must be taken into account when comparing the results of FAF imaging obtained from different cSLO devices. ${ }^{3}$

Numerous studies suggest that FAF imaging could assist the detection and/or monitoring of various retinal conditions, ${ }^{1,3}$ for example, geographic atrophy, ${ }^{4,6-12}$ choroidal neovascularisation development in age-related macular degeneration (AMD), ${ }^{13-17}$ retinal pigment epithelium alterations, ${ }^{14,18-22}$ retinitis pigmentosa, ${ }^{7,23-30}$ Best's disease, 2,31-34 Stargardt disease, 2,34-37 cystoid macular oedema, ${ }^{38-43}$ diabetic macular oedema, ${ }^{44-49}$ and central serous chorioretinopathy, ${ }^{14,50-58}$ among others.

Assessment of FAF has also been specified as an outcome in clinical research studies of therapies for retinal conditions, for example, with anti-vascular endothelial growth factor drugs, ${ }^{18,59-61}$ other drugs, $, 962,63$ photodynamic therapy, ${ }^{64}$ and vitrectomy. ${ }^{65}$ However, despite this extensive interest in FAF imaging, these studies have not hitherto been critically assessed and it is unclear how much robust quantitative evidence exists to support the accuracy of FAF imaging using cSLO, that is, its sensitivity and specificity, for the diagnosis and/or monitoring of retinal conditions.

To determine the accuracy of FAF imaging using cSLO for the diagnosis and/or monitoring of retinal conditions, we conducted a systematic review of studies in a wide range of retinal conditions. Full details of the review are reported by Frampton et al. ${ }^{66}$ Here, we summarise the key methods and findings of the systematic review, and a review update conducted in October 2016, and we consider the implications of the current evidence for the application of FAF imaging to detect and/or monitor retinal conditions in clinical practice.

\section{Materials and methods}

The methods for this systematic review were specified a priori in a protocol ${ }^{67}$ that was subjected to independent peer review.

\section{Identification of evidence}

A comprehensive search strategy was developed and refined by an experienced information specialist to identify studies on the diagnosis and/or monitoring of retinal conditions based on the following pre-specified inclusion and exclusion criteria:

- Population: of any age with any retinal condition, excluding those resulting from malignancy, or those secondary to major ocular conditions (eg, glaucoma) or trauma.

- Index test: FAF imaging performed using cSLO.

- Reference standard: fundus imaging performed using any clinically relevant method (eg, fundus photography, fundus fluorescein angiography, indocyanine green angiography, optical coherence tomography, or any combination of relevant tests).

- Primary outcomes: sensitivity and/or specificity for the diagnosis or monitoring of retinal conditions.

- Secondary outcomes (applicable only if primary outcomes were also reported): inter- and intra-observer agreement, adverse events, test acceptability to patients and clinicians, and test interpretability.

- Study designs: any study design, provided that the index test(s) and reference standard were compared in the same patient group and the sample size was $\geq 10$ eyes per group.

Searches were conducted in the following electronic bibliographic databases: MEDLINE; PreMedline InProcess and Other Non-Indexed Citations; EMBASE; the Cochrane Library; Web of Science, including the Science Citation Index-Expanded (SCI-E) and the Conference Proceedings Citation Index-Science (CPCI-S); Database of Abstracts of Reviews of Effectiveness (DARE); Health Technology Assessment Database (HTA); NHS Economic Evaluation Database (NHSEED); and the MEDION database of diagnostic accuracy studies. In addition, searches were made in internet pages of the American Academy of Ophthalmology (AAO); Association for Research in Vision and Ophthalmology 
(ARVO); Cochrane Eyes and Vision Group (CEVG); European Association for Vision and Eye Research (EVER); and the Royal College of Ophthalmologists. Trial registries searched were the Cochrane Central Register of Controlled Trials, UK Clinical Research Network Portfolio Database; World Health Organization International Clinical Trials Registry Platform (WHO ICTRP); Current Controlled Trials; Clinical Trials.gov; and NIHR Clinical Research Network Portfolio. In addition, bibliographies of retrieved papers and relevant systematic reviews were searched. All searches were conducted for the period from 1990 ( 10 years prior to the likely publication of the earliest relevant evidence) to November 2014 and searches were limited to the English language.

\section{Search update}

The current paper was submitted for publication 20 months after our original literature search. To check whether our conclusions remain applicable, we conducted an updated search in October 2016. Limited team availability precluded us re-running the full searches described above. Instead, we re-ran our original MEDLINE search. ${ }^{66}$ and supplemented this with a broad search in Google Scholar using the free text term 'fundus autofluorescence imaging'. The first 200 potentially relevant references identified in Google Scholar, as judged by one reviewer against the inclusion criteria, and all references identified in the MEDLINE search were imported into an Endnote library. As there were differences in the methods of the original and update searches, we present the results obtained from the two searches separately below.

\section{Selection of evidence}

Titles and abstracts identified in the original searches were screened independently by two reviewers against the inclusion criteria to identify potentially relevant bibliographic records. Full-text articles were retrieved for those records judged to be relevant or unclear at the title and abstract screening step. Full-text articles were screened by one reviewer and checked by a second reviewer. At both steps of the selection process, a standard study selection worksheet listing the inclusion criteria was employed, ${ }^{66}$ and any disagreements were resolved by discussion among the two reviewers or, if necessary, by involving a third reviewer. If a study was reported in more than one article at the full-text

assessment step, all the articles relating to the study were grouped together for the eligibility assessment.

The study selection process for the search update was identical to that of the original search except that the title and abstract screening step was conducted by one reviewer. A randomly selected subset of $20 \%$ of the titles and abstracts identified in the search update was checked by a second reviewer, with $100 \%$ reviewer agreement (Supplementary Appendix A).

\section{Data extraction, critical appraisal, and data synthesis}

The data extraction, critical appraisal, and data synthesis steps of the original systematic review were conducted by one reviewer and checked by a second reviewer. Any disagreements were resolved through discussion between the reviewers. For the review update, these steps were conducted by one reviewer. The update search results are illustrative and as such are reported separately from those of the original review below.

Data were extracted from the included studies using a pre-designed and pilot-tested data extraction form ${ }^{66}$ to minimise errors. If test accuracy outcomes were not fully reported, missing values were calculated by the reviewers where possible.

Methodological rigour of the included studies was assessed using the Cochrane Collaboration adaptation ${ }^{68}$ of the QUADAS tool, ${ }^{69}$ which asks 11 questions about the characteristics of the primary studies. These questions aim to identify potential threats to the validity of the study findings and (in our interpretation) reflect 10 different types of bias that may occur in studies of test accuracy. We have interpreted the QUADAS questions to determine whether for each of the included studies there is a low, high, or unclear risk of each type of bias (Supplementary Table 1 provides an explanation of the QUADAS questions and the types of bias being assessed).

The method of data synthesis allowed for meta-analysis of test accuracy outcomes if studies were sufficiently similar, as specified in the review protocol. ${ }^{67}$

\section{Results}

As there were some differences in the methods of our original systematic review and the review update, results of the review update are presented separately below.

\section{Identification and characteristics of studies}

Searches conducted in November 2014 yielded 2240 unique bibliographic records, of which 2034 (91\%) were excluded upon inspection of the title and abstract (Figure 1). Of 206 bibliographic records obtained for further scrutiny, 198 (96\%) were excluded, primarily because 163 (85\%) did not report (or provide sufficient data to enable us to calculate) sensitivity and/or specificity of FAF imaging. Eight research publications reporting eight unique studies met the eligibility criteria and were included in the systematic review. $39,41,48,70-74$ 


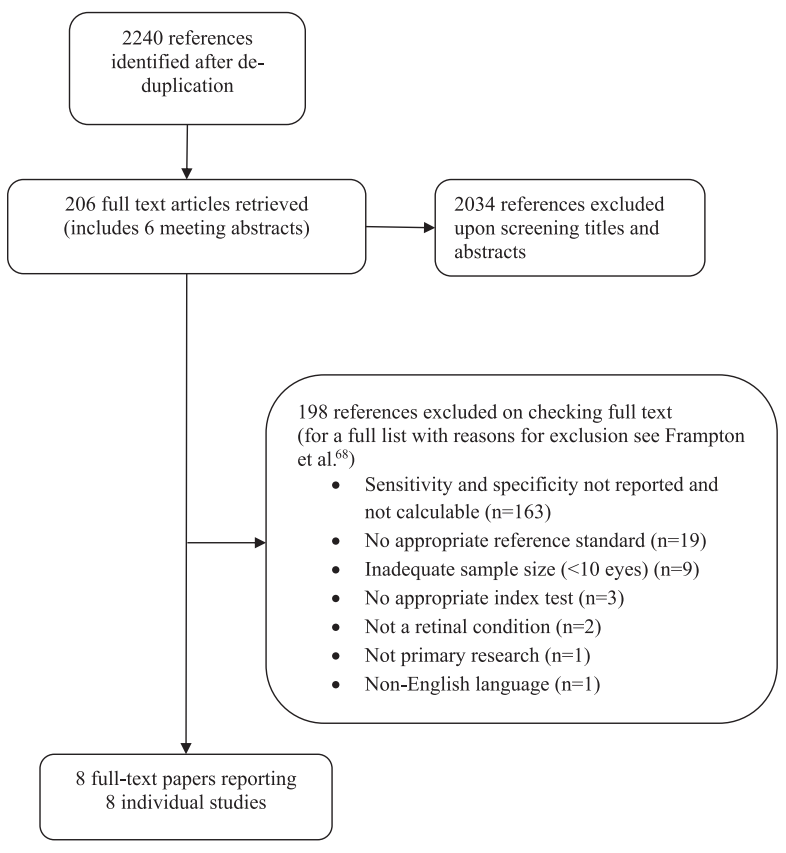

Figure 1 Flow chart for the identification of studies.

Key characteristics of the included studies are summarised in Supplementary Table 2 and are given in detail in the full study report. ${ }^{66}$ Although FAF imaging may be used to detect autofluorescence in a wide range of retinal conditions, the eight included studies cover only choroidal neovascularisation in $\mathrm{AMD},{ }^{70}$ reticular pseudodrusen in AMD, ${ }^{71-73}$ cystoid macular oedema, ${ }^{39,41}$ and diabetic macular oedema. ${ }^{48,74}$ Six of these studies were European, one was conducted in the United States, ${ }^{72}$ and one in Japan. ${ }^{73}$ The number of participants ranged from 34 to 138 and the number of eyes from 34 to 263 . None of the studies involved people younger than 17 years of age, and the mean age ranged from 49 to 76 years, although not all studies gave this information. The studies differed in whether they admitted participants with any ocular comorbidity besides the retinal condition of primary interest. Despite the extensive research interest noted above in the use of FAF imaging for monitoring the course of retinal conditions and their response to therapy, none of the included studies investigated FAF imaging for monitoring disease progression or response to therapy. The study designs were either prospective or retrospective cohorts, and in all cases the unit of analysis was the eye. However, the studies differed according to whether they included one eye per patient, both eyes, or a mixture of single eyes and both eyes (Supplementary Table 3). Furthermore, the studies on reticular pseudodrusen in $\mathrm{AMD}^{71-73}$ differed according to the stage of AMD, and whether it affected one or both eyes; the studies on cystoid macular oedema ${ }^{39,41}$ differed in the primary conditions from which the cystoid macular oedema developed; and the studies on diabetic macular oedema ${ }^{48,74}$ differed as to whether they included ocular comorbidities. Because of this extensive heterogeneity among the studies, we considered it inappropriate to conduct a meta-analysis.

The studies (where reported) used variants of the HRA cSLO for obtaining FAF images (ie, the index test), primarily employing an excitation wavelength of $488 \mathrm{~nm}$ (blue), with two studies additionally including a $514 \mathrm{~nm}$ (green) wavelength ${ }^{48}$ and a $790 \mathrm{~nm}$ (near-infrared) wavelength ${ }^{73}$ (Table 1). The comparator tests (ie, reference standards) included various types of colour fundus photography, fluorescein angiography, scanning laser ophthalmoscopy, and optical coherence tomography, although two studies ${ }^{71,73}$ employed comparisons in which a positive result was required on one of five ${ }^{71}$ or two of seven ${ }^{73}$ imaging tests. The diagnostic criteria on FAF imaging for each of the retinal conditions were qualitative (ie, descriptive) in all studies, and only one of the studies reported an objective (quantitative) approach for determining how abnormal (hypo or hyper) autofluorescence was defined. ${ }^{72}$ The three studies on reticular pseudodrusen all specified that the diagnostic criterion was a reticular pattern of hypo-autofluorescence against a background of mildly increased autofluorescence, although there were differences in how this was described in each study. ${ }^{71-73}$ The two studies on cystoid macular oedema ${ }^{39,41}$ both appeared to diagnose the condition as round or oval patterns of autofluorescence at the fovea, although this was not stated explicitly in one study. ${ }^{39}$ The two studies on diabetic macular oedema ${ }^{48,74}$ only mentioned the diagnostic criteria briefly and differed in how these were described.

\section{Risks of bias}

Assessment of the risk of bias (Table 2) identified two main problems with the studies (the decision criteria are shown in Supplementary Table 1). First, seven studies were deemed to be at high risk and one at unclear risk of spectrum bias, since the population with retinal disease was not representative of those who would receive a FAF imaging test in clinical practice. Two studies on reticular pseudodrusen in AMD were also deemed to be at high risk of biases related to the use of an inappropriate reference standard (differential verification bias and incorporation bias). ${ }^{71,73}$ The second problem is that, for many domains of bias that were assessed, the risk of bias was unclear due to a lack of adequate reported methodological information (unclear risk of bias means that the possibility of high risk of bias could not be ruled out). Notably, all the studies were deemed to be at unclear 


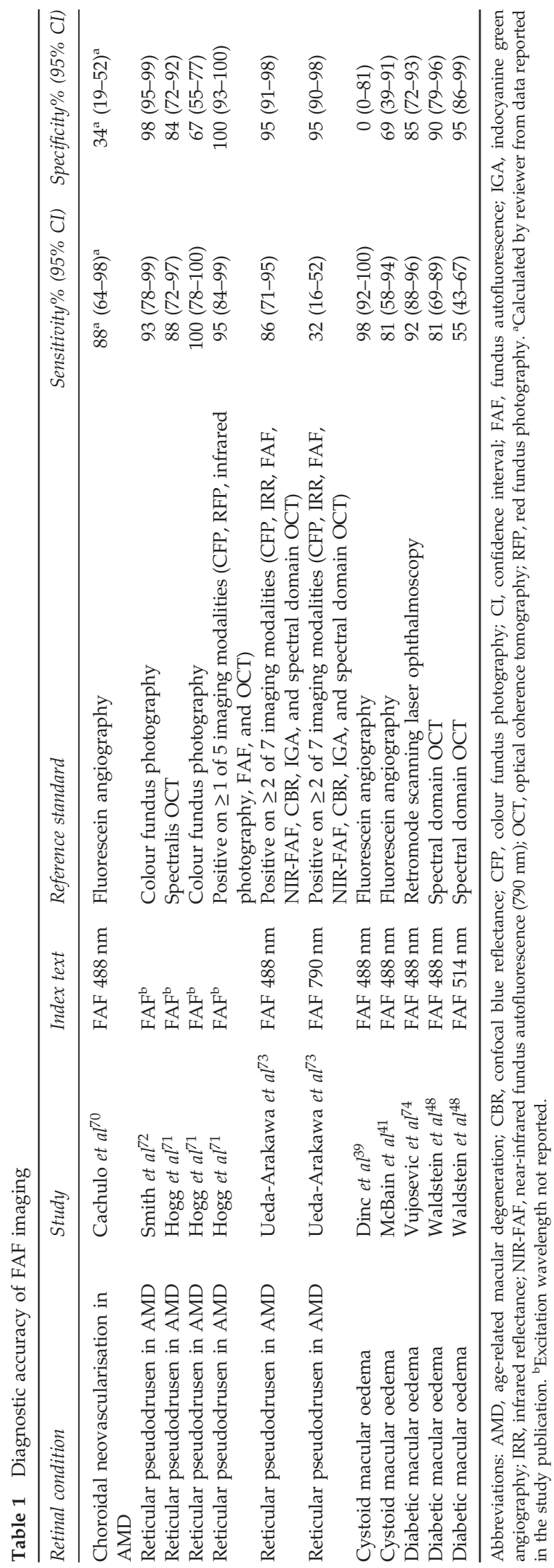



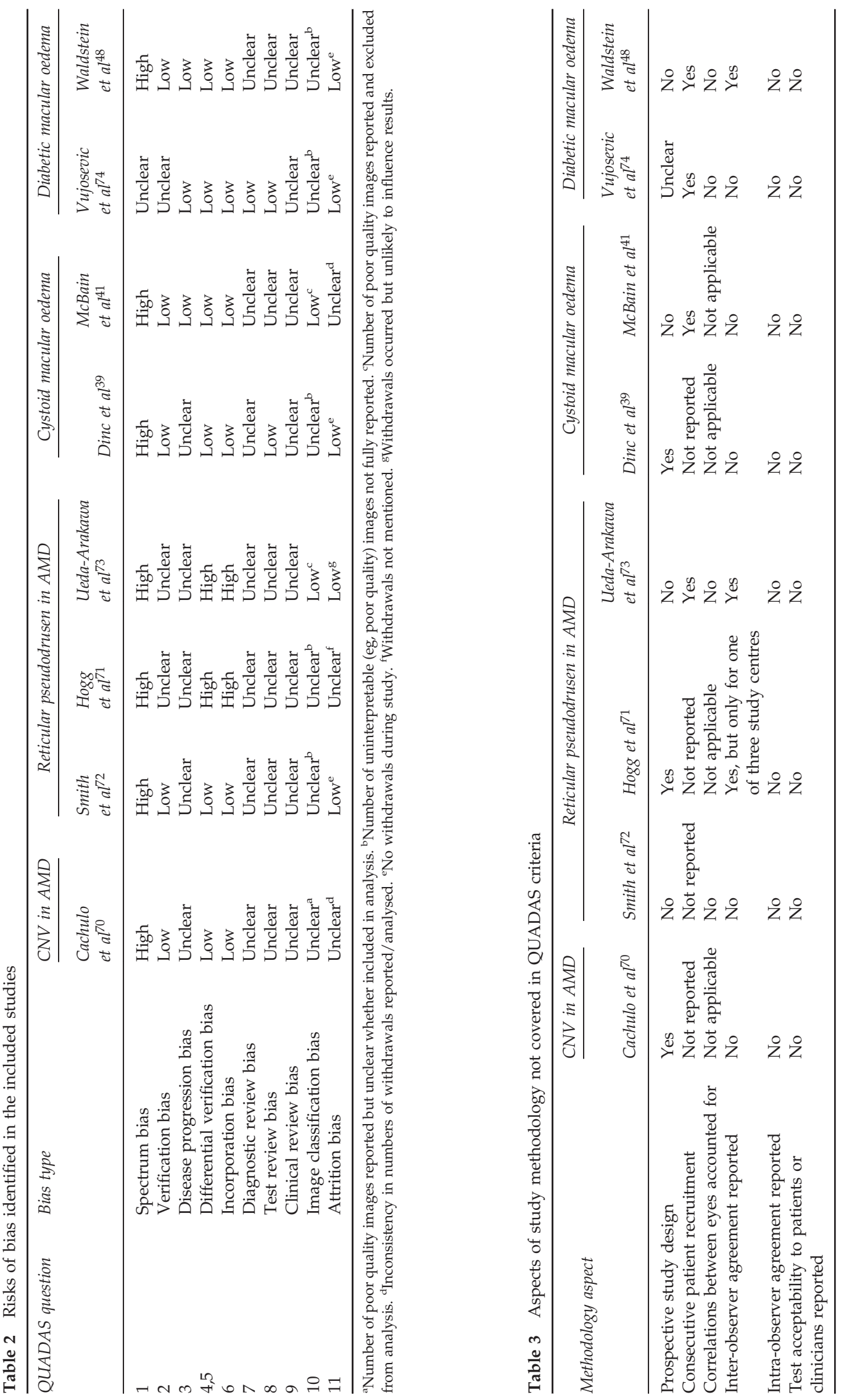
risk of clinical review bias since none of them provided unequivocal statements about which clinical information, if any, was considered when interpreting the FAF images. In clinical practice, it is unlikely that FAF images would be interpreted in isolation of other clinical information, so it is possible that these studies may have systematically overestimated or underestimated the sensitivity and specificity of FAF imaging relative to how it would be applied in clinical practice.

\section{Other aspects of study methodology}

Several aspects of the study methodology that are not covered by QUADAS criteria could have implications for interpretation of the study findings (Table 3). Ideally, to minimise the risk of selection bias, the cohort studies should have been conducted prospectively and should have recruited participants consecutively. ${ }^{75}$ However, as shown in Table 3, this is not clear in the publications of five of the eight studies and adds further uncertainty about the reliability of these studies' findings.

Four of the eight reviewed studies analysed both of the participants' eyes, and in such cases it would be appropriate to consider intra-subject correlations in the analysis. ${ }^{76}$ However, these studies did not mention the correlation or make any adjustment for it. Although it is not clear that this would lead to bias, intra-individual correlation could lead to underestimation of the standard error, and hence the confidence intervals, for sensitivity and specificity estimates.

Despite the subjective nature of the diagnostic criteria, only three of the eight studies investigated inter-grader agreement in the classification of retinal images. Limited information was provided about the expertise of the image graders, who were described as two independent ophthalmologists, ${ }^{73}$ two retinal specialists trained in image grading, ${ }^{74}$ and two independent graders. ${ }^{48}$ None of the studies reported intra-grader agreement (ie, a person's consistency of image interpretation), or whether the FAF imaging technique was considered acceptable by the participants or clinicians.

\section{Accuracy of FAF imaging}

As shown in Table 1, diagnostic sensitivity of FAF imaging compared to various reference standards ranged from 32 to $100 \%$ and specificity from 34 to $100 \%$. The sensitivity was lowest when FAF emission wavelengths longer than $488 \mathrm{~nm}$ were used (32\% for $790 \mathrm{~nm}^{73}$ and $55 \%$ for $514 \mathrm{~nm}^{48}$ ), although two studies with relatively good sensitivity $(88-100 \%)$ did not specify the emission wavelengths they employed. ${ }^{71,72}$ Specificity was lowest (34\%) for the study on choroidal neovascularisation in $\mathrm{AMD}^{70}$ and one study on cystoid macular oedema $(0 \%) .{ }^{39}$ In the latter study, specificity was zero since no true- negative results were identified when FAF imaging was compared against fluorescein angiography. ${ }^{39}$ However, it is questionable whether any of these test accuracy findings are clinically meaningful, given their extensive methodological limitations, risks of bias, and the interpretational uncertainties mentioned above.

\section{Results of the review update}

Supplementary Appendix A provides results of the updated search in October 2016, including a PRISMA flow chart showing the study selection process; a list of studies excluded at full-text screening; the QUADAS risk of bias assessment; and an overview of the characteristics and results of the included studies. After removing duplicates, the updated search in October 2016 yielded 380 new references. Eighteen references were retrieved for screening on full text, ${ }^{77-94}$ of which four met the criteria for inclusion in the systematic review. ${ }^{79,80,82,91}$

The four new references reported four unique studies. These investigated FAF imaging accuracy for detecting: reticular pseudodrusen in AMD (two studies ${ }^{80,91}$ ); hydroxychloroquine-induced retinopathy (one study ${ }^{79}$ ); and lacquer cracks in highly myopic eyes (one study ${ }^{82}$ ). In two of these studies, the FAF imaging excitation wavelength was $488 \mathrm{~nm} ;{ }^{79,80}$ in one study, both 488 and $787 \mathrm{~nm}$ (near-infrared) wavelengths were employed; and one study did not report the excitation wavelength. ${ }^{91}$ The reference standards were: spectral domain optical coherence tomography in addition to one or more other imaging methods to detect reticular pseudodrusen; 80,91 multifocal electroretinography to detect hydroxychloroquine-induced retinopathy; ${ }^{79}$ and indocyanine green angiography to detect retinal lacquer cracks. ${ }^{82}$ In all studies, the unit of analysis was the eye and sample sizes ranged from 56 eyes $^{82}$ to 600 eyes. $^{91}$

Critical appraisal of the four new studies indicated that they exhibited similar methodological limitations to the studies included in our original review: all four studies were deemed to be at high risk of spectrum bias because they excluded patients with comorbidities and therefore would not be reflective of the case mix presenting in clinical practice; and all four studies were deemed to be at unclear risk of clinical review bias because it was not clear whether the clinical information available to FAF image readers was the same as that which would be available in clinical practice. The studies were judged to be at low or unclear risks of the other types of bias assessed by the QUADAS instrument.

Sensitivity of FAF imaging across these retinal conditions was: reticular pseudodrusen: $73^{80}-89 \% ;{ }^{91}$ hydroxychloroquine-induced retinopathy: $80 \%, 79$ and lacquer cracks: $12.5 \%$ with $488 \mathrm{~nm}$ FAF and $93 \%$ with 787 nm FAF. ${ }^{82}$ Specificity was $97-100 \%$ for reticular 
pseudodrusen 80,91 and $88 \%$ for hydroxychlorine retinopathy, ${ }^{79}$ but not reported for retinal lacquer cracks. ${ }^{82}$ None of the four studies reported sufficient data for true-positive, true-negative, false-positive, or falsenegative test results, or predictive values, to be calculated.

\section{Discussion}

Our systematic review highlights some key issues concerning the use of FAF imaging for detecting or monitoring retinal conditions in clinical practice. The academic literature shows that FAF imaging is used extensively for the detection and/or monitoring of a wide range of retinal conditions in research studies, but our critical examination of the evidence identified that only a minority of these studies have formally assessed the accuracy of the method, for very few retinal conditions. Moreover, despite the relatively high estimates of sensitivity and/or specificity reported in some studies, the methodological shortcomings of the studies, together with inadequate reporting of study details, leave us uncertain as to whether the results of this limited set of primary studies are reliable. It is also unclear whether the results of any of these research studies would be truly reflective of how FAF imaging tests would be interpreted if applied in clinical practice.

FAF imaging could have potential for diagnosing inherited retinal dystrophies such as retinitis pigmentosa, cone-rod dystrophies, and Stargardt disease, or for monitoring the progression of Best's disease, ${ }^{32}$ but our original systematic review and the update conducted in October 2016 did not identify any quantitative comparisons of test accuracy in these conditions.

In the present systematic review, we have followed the paradigm of test accuracy assessment, ${ }^{68,95,96}$ in which we have quantified the accuracy of FAF imaging (the index test) when compared against a comparator (the reference standard). The paradigm of test accuracy assessment requires that the reference standard should, preferably, be the 'gold standard', that is, identifying with optimal accuracy (ideally $100 \%$ ) which of the patients tested have a specified retinal condition (true positives) and which do not have the condition (true negatives). However, since FAF imaging based on cSLO utilises confocal optics and filters to reduce optical artefacts and provide a highresolution image, it is plausible that FAF imaging would be more accurate than existing imaging methods (eg, colour fundus photography) at detecting certain types of retinal pathology. ${ }^{97}$ FAF imaging also detects different retinal processes compared to other imaging methods; for example, while fluorescein angiography detects leakage, FAF imaging detects metabolic activity. ${ }^{97}$ For these reasons, there may not always be an obvious gold standard test against which to compare FAF imaging. Publications that have discussed the clinical relevance of
FAF imaging $4,97,98$ suggest that the benefits of FAF imaging have largely been inferred from descriptive comparisons of the FAF patterns seen in diseased eyes against those seen in normal eyes rather than by involving quantitative comparisons against a gold standard test. The use of FAF imaging for monitoring the extent of geographic atrophy, $4,6-12$ and for monitoring the presence and extent of the 'autofluorescent ring' of retinitis pigmentosa, ${ }^{7,23-30}$ are examples of where there is expert consensus that characteristic patterns of FAF are associated with disease progression, yet this has not been established by quantitative comparisons of imaging methods (other than correlations between FAF intensity and the spatial distribution of retinal pathology). Currently, there is no universally accepted standard approach for quantifying FAF, ${ }^{4,72}$ although image grading software has been developed that can quantify the local or general background intensity of the greyscale FAF image obtained from cSLO, so as to identify which pixels could be classified as indicating hypo- or hyperautofluorescence. ${ }^{72}$ For FAF imaging to be used in clinical ophthalmology in a consistent way, it would be necessary to determine and agree on thresholds for increased or decreased FAF (ie, pixel intensity) that can be reliably considered as abnormal for different retinal conditions.

\section{The position of FAF imaging in the care pathway}

A new test such as FAF imaging could have one of three main roles: triage, add-on, or replacement. ${ }^{99}$ In 5 of the total 12 studies that were included when considering both the original review and the review update, the aim was primarily to explore relationships between FAF and retinal morphological characteristics rather than to compare the accuracy of imaging tests. ${ }^{70-72,79,80}$ One study specifically asked whether FAF imaging could be an ancillary test, for detecting cystoid macular oedema, ${ }^{39}$ while one study specifically compared FAF imaging as an index test against fluorescein angiography as the reference standard for diagnosing cystoid macular oedema, ${ }^{41}$ although the authors did not explicitly discuss whether FAF imaging could replace angiography. The authors of several studies suggested that FAF imaging could assist diagnosis of retinal conditions as one of the several imaging modalities (ie, as an additional rather than a replacement test), for reticular pseudodrusen in $\mathrm{AMD},{ }^{73,80,91}$ diabetic macular oedema, ${ }^{48,74}$ hydroxychloroquine-induced retinal toxicity, ${ }^{79}$ and retinal lacquer cracks. ${ }^{82}$

When interpreting the studies on FAF imaging, it is important to keep in mind that finding out whether a test can serve its role is not exclusively based on sensitivity and specificity of the test alone, but also on how the accuracy of the existing testing pathway is changed by the test. ${ }^{99}$ Although most of the studies included in our 
systematic review suggest that FAF imaging has potential as an add-on test, alongside other retinal imaging methods (eg, optical coherence tomography, plus other tests such as angiography or fundus photography as needed), none of the studies calculated the diagnostic accuracy of a multiple-test strategy.

\section{Could FAF imaging replace fluorescein or indocyanine green angiography?}

Given that FAF imaging is non-invasive, there might be benefits to both patients and the health service if FAF imaging could, in some cases, replace retinal angiography, which is the most frequently used invasive retinal imaging test. Retinal angiography carries a risk of complications, including adverse hypersensitivity reactions to dye, ranging from mild nausea or hives to (less commonly) anaphylaxis and death. It is also contradicted in pregnancy, and requires specialist clinical facilities, including a sterile clinical environment and staff qualified in venous access. ${ }^{39}$ The extent to which FAF imaging might replace retinal angiography would likely depend on the pathological process of the retinal disease. For example, angiography is valuable in AMD and macular oedemas for detecting fluid perfusion and leakage that might not be detectable using FAF imaging. However, of the four studies that employed angiography as a reference standard, none tested whether FAF imaging could replace fluorescein angiography ${ }^{39,41,70}$ or indocyanine green angiography. ${ }^{79}$ These studies also did not report patients' perceptions of the test procedures or whether or not the angiography reference standard was associated with any adverse events. Further evidence would be helpful to clarify the magnitude of benefits or disadvantages to patients and health services of any switch from fluorescein angiography to FAF imaging.

\section{Quality assurance of FAF imaging}

A problem in clinical ophthalmology is that formal training and clinical guidelines regarding the use of new imaging techniques in diagnosing and monitoring various ocular conditions is currently lacking. ${ }^{100}$ It is important that clinicians (as well as researchers) using cSLO for FAF imaging are aware of the limitations of the method, and receive adequate training in how to operate the equipment in a standardised way and in how to interpret the resulting FAF images. Despite subjectivity in the interpretation of FAF images, ${ }^{79}$ our systematic review indicates that these quality assurance steps are rarely considered in research studies, so the reliability of FAF image interpretation when compared both between image graders and for the same grader at different times is largely unknown.

\section{Limitations of the review}

Our systematic review has three potential limitations. First, for practical reasons, we limited searches to studies published in English. We cannot rule out that relevant studies might have been published in other languages. Second, we did not contact authors to clarify any missing or unclear information in the included studies. However, we believe it unlikely that this would have affected our conclusions since major limitations (eg, inappropriate reference standards and/or lack of clinical relevance) were evident in most of the studies based on the peerreviewed information that was available. Third, to keep the review manageable, we excluded retinal conditions that resulted from malignancy, trauma, or were secondary to other ocular diseases (such as glaucoma). The scope of our conclusions therefore excludes studies on these particular conditions.

\section{Conclusion and recommendations}

In conclusion, the scientific literature identified in our searches demonstrates that there is considerable interest in the use of FAF imaging for characterising and describing retinal conditions; however, only a small proportion of the literature met our inclusion criteria, indicating a lack of quantitative assessments of the accuracy of the method for diagnosis or monitoring. We have identified a need for prospective quantitative research into the accuracy of FAF imaging, particularly in the inherited retinal dystrophies, geographic atrophy, and central serous chorioretinopathy; these are important retinal conditions for which we identified no quantitative test accuracy evidence. Our systematic review also highlights that there is a need for improved reporting of research on FAF imaging. In particular, future research studies should report:

- which information, if any, alongside the results of FAF imaging tests is used to inform diagnostic or other clinical decisions, so as to clarify whether test results are being interpreted in the research studies in the same way that they would be in clinical practice;

- the training and experience of image graders;

- inter-grader and intra-grader agreement in classification of FAF images;

- any resources associated with the acquisition, processing, quality assurance, and interpretation of FAF images-this would help to clarify the pros and cons of FAF imaging relative to other imaging modalities (and would also assist any evaluation of the costeffectiveness of FAF imaging that are conducted). 


\section{Conflict of interest}

The authors declare no conflict of interest.

\section{Acknowledgements}

This project was funded by the National Institute for Health Research (NIHR) Health Technology Assessment (HTA) programme (project number 14/151/02) and is published in full in the Health Technology Assessment journal. This report presents independent research commissioned by the NIHR. The views and opinions expressed by the authors in this publication are those of the authors and do not necessarily reflect those of the HTA programme, NIHR, or the Department of Health. We thank Karen Welch, information scientist, for conducting the systematic review searches. The systematic review supporting this publication is registered in the PROSPERO database, reference CRD42014014997.

\section{References}

1 Lois N, Forrester V. Fundus Autofluorescence 1st Ed. Lippincot Williams and Wilkins: Philadelphia, PA, USA, 2009.

2 Boon CJF, Klevering BJ, Keunen JEE, Hoyng CB, Theelen T. Fundus autofluorescence imaging of retinal dystrophies. Vision Res 2008; 48: 2569-2577.

3 Schmitz-Valckenberg S, Holz FG, Bird AC, Spaide RF. Fundus autofluorescence imaging: review and perspectives. Retina 2008; 28(3): 385-409.

4 Sepah YJ, Akhtar A, Sadiq MA, Hafeez Y, Nasir H, Perez B et al. Fundus autofluorescence imaging: fundamentals and clinical relevance. Saudi J Ophthalmol 2014; 28: 111-116.

5 Webb RH, Hughes GW, Delori FC. Confocal scanning laser ophthalmoscope. Appl Opt 1987; 26: 1492-1499.

6 Bearelly S, Cousins SW. Fundus autofluorescence imaging in age-related macular degeneration and geographic atrophy. Adv Exp Med Biol 2010; 664: 395-402.

7 Jeong YJ, Hong IH, Chung JK, Kim KL, Kim HK, Park SP. Predictors for the progression of geographic atrophy in patients with age-related macular degeneration: fundus autofluorescence study with modified fundus camera. Eye 2014; 28(2): 209-218.

8 Lee JY, Lee DH, Lee JY, Yoon YH. Correlation between subfoveal choroidal thickness and the severity or progression of nonexudative age-related macular degeneration. Invest Ophthalmol Vis Sci 2013; 54(12): 7812-7818.

9 Petrou PA, Cunningham D, Shimel K, Harrington M, Hammel K, Cukras CA et al. Intravitreal sirolimus for the treatment of geographic atrophy: results of a phase I/II clinical trial. Invest Ophthalmol Vis Sci 2014; 56(1): 330-338.

10 Pilotto E, Guidolin F, Convento E, Spedicato L, Vujosevic S, Cavarzeran $\mathrm{F}$ et al. Fundus autofluorescence and microperimetry in progressing geographic atrophy secondary to age-related macular degeneration. $\mathrm{Br} \mathrm{J}$ Ophthalmol 2013; 97(5): 622-626.
11 Schmitz-Valckenberg S, Bindewald-Wittich A, DolarSzczasny J, Dreyhaupt J, Wolf S, Scholl HP et al. Correlation between the area of increased autofluorescence surrounding geographic atrophy and disease progression in patients with AMD. Invest Ophthalmol Vis Sci 2006; 47(6): 2648-2654.

12 Simader C, Sayegh RG, Montuoro A, Azhary M, Koth AL, Baratsits $\mathrm{M}$ et al. A longitudinal comparison of spectraldomain optical coherence tomography and fundus autofluorescence in geographic atrophy. Am J Ophthalmol 2014; 158(3): 557-566.

13 Batioglu F, Demirel S, Ozmert E, Oguz YG, Ozyol P. Autofluorescence patterns as a predictive factor for neovascularization. Optom Vis Sci 2014; 91(8): 950-955.

14 Cuba J, Gomez-Ulla F. Fundus autofluorescence: applications and perspectives. Arch Soc Esp Oftalmol 2013; 88(2): 50-55.

15 Dandekar SS, Jenkins SA, Peto T, Scholl HP, Sehmi KS, Fitzke FW et al. Autofluorescence imaging of choroidal neovascularization due to age-related macular degeneration. Arch Ophthalmol 2005; 123(11): 1507-1513.

16 Toju R, Iida T, Sekiryu T, Saito M, Maruko I, Kano M. Nearinfrared autofluorescence in patients with idiopathic submacular choroidal neovascularization. Am J Ophthalmol 2012; 153(2): 314-319.

17 Vaclavik V, Vujosevic S, Dandekar SS, Bunce C, Peto T, Bird AC. Autofluorescence imaging in age-related macular degeneration complicated by choroidal neovascularization: a prospective study. Ophthalmology 2008; 115(2): 342-346.

18 Asao K, Gomi F, Sawa M, Nishida K. Additional antivascular endothelial growth factor therapy for eyes with a retinal pigment epithelial tear after the initial therapy. Retina 2014; 34(3): 512-518.

19 Clemens CR, Alten F, Baumgart C, Heiduschka P, Eter N. Quantification of retinal pigment epithelium tear area in age-related macular degeneration. Retina 2014; 34(1): 24-31.

20 Kellner U, Kellner S, Weinitz S. Fundus autofluorescence (488 NM) and near-infrared autofluorescence (787 NM) visualize different retinal pigment epithelium alterations in patients with age-related macular degeneration. Retina 2010; 30(1): 6-15.

21 Kramer M, Priel E. Fundus autofluorescence imaging in multifocal choroiditis: beyond the spots. Ocul Immunol Inflamm 2014; 22(5): 349-355.

22 Kumar N, Mrejen S, Fung AT, Marsiglia M, Loh BK, Spaide RF. Retinal pigment epithelial cell loss assessed by fundus autofluorescence imaging in neovascular age-related macular degeneration. Ophthalmology 2013; 120(2): 334-341.

23 Aizawa S, Mitamura Y, Hagiwara A, Sugawara T, Yamamoto S. Changes of fundus autofluorescence, photoreceptor inner and outer segment junction line, and visual function in patients with retinitis pigmentosa. Clin Exp Optom 2010; 38(6): 597-604.

24 Iriyama A, Yanagi Y. Fundus autofluorescence and retinal structure as determined by spectral domain optical coherence tomography, and retinal function in retinitis pigmentosa. Graefes Arch Clin Exp Ophthalmol 2012; 250(3): 333-339.

25 Lenassi E, Troeger E, Wilke R, Hawlina M. Correlation between macular morphology and sensitivity in patients with retinitis pigmentosa and hyperautofluorescent ring. Invest Ophthalmol Vis Sci 2012; 53(1): 47-52.

26 Lima LH, Burke T, Greenstein VC, Chou CL, Cella W, Yannuzzi LA et al. Progressive constriction of the 
hyperautofluorescent ring in retinitis pigmentosa. Am J Ophthalmol 2012; 153(4): 718-727.

27 Murakami T, Akimoto M, Ooto S, Suzuki T, Ikeda H, Kawagoe $\mathrm{N}$ et al. Association between abnormal autofluorescence and photoreceptor disorganization in retinitis pigmentosa. Am J Ophthalmol 2008; 145(4): 687-694.

28 Robson AG, Lenassi E, Saihan Z, Luong VA, Fitzke FW, Holder GE et al. Comparison of fundus autofluorescence with photopic and scotopic fine matrix mapping in patients with retinitis pigmentosa: 4- to 8-year follow-up. Invest Ophthalmol Vis Sci 2012; 53(10): 6187-6195.

29 Robson AG, Tufail A, Fitzke F, Bird AC, Moore AT, Holder GE et al. Serial imaging and structure-function correlates of high-density rings of fundus autofluorescence in retinitis pigmentosa. Retina 2011; 31(8): 1670-1679.

30 Wakabayashi T, Sawa M, Gomi F, Tsujikawa M. Correlation of fundus autofluorescence with photoreceptor morphology and functional changes in eyes with retinitis pigmentosa. Acta Opthalmol 2010; 88(5): e177-e183.

31 Duncker T, Greenberg JP, Ramachandran R, Hood DC, Smith RT, Hirose T et al. Quantitative fundus autofluorescence and optical coherence tomography in best vitelliform macular dystrophy. Invest Ophthalmol Vis Sci 2014; 55(3): 1471-1482.

32 Jarc-Vidmar M, Kraut A, Hawlina M. Fundus autofluorescence imaging in Best's vitelliform dystrophy. Klin Monbl Augenheilkd 2003; 220(12): 861-867.

33 Querques G, Zerbib J, Georges A, Massamba N, Forte R, Querques $\mathrm{L}$ et al. Multimodal analysis of the progression of Best vitelliform macular dystrophy. Mol Vis 2014; 20: 575-592.

34 von Ruckmann A, Fitzke FW, Bird AC. In vivo fundus auto fluorescence in macular dystrophies. Arch Ophthalmol 1997; 115(5): 609-615.

35 Chen B, Tosha C, Gorin MB, Nusinowitz S. Analysis of autofluorescent retinal images and measurement of atrophic lesion growth in Stargardt disease. Exp Eye Res 2010; 91(2): 143-152.

36 North V, Gelman R, Tsang SH. Juvenile-onset macular degeneration and allied disorders. Dev Ophthalmol 2014; 53: 44-52.

37 Pichi F, Morara M, Veronese C, Nucci P, Ciardella AP. Multimodal imaging in hereditary retinal diseases. I Ophthalmol 2013; 2013: 634351.

38 Bessho K, Gomi F, Harino S, Sawa M, Sayanagi K, Tsujikawa $\mathrm{M}$ et al. Macular autofluorescence in eyes with cystoid macula edema, detected with $488 \mathrm{~nm}$-excitation but not with $580 \mathrm{~nm}$-excitation. Graefes Arch Clin Exp Ophthalmol 2009; 247(6): 729-734.

39 Dinc UA, Tatlipinar S, Yenerel M, Gorgun E, Ciftci F. Fundus autofluorescence in cystoid macular edema: can it be an ancillary test? Retina 2010; 18(1): 12-17.

40 Kellner S, Weinitz S, Farmand G, Kellner U. Cystoid macular oedema and epiretinal membrane formation during progression of chloroquine retinopathy after drug cessation. Br J Ophthalmol 2014; 98(2): 200-206.

41 McBain VA, Forrester JV, Lois N. Fundus autofluorescence in the diagnosis of cystoid macular oedema. Br J Ophthalmol 2008; 92(7): 946-949.

42 Pece A, Isola V, Holz F, Milani P, Brancato R. Autofluorescence imaging of cystoid macular edema in diabetic retinopathy. Ophthalmologica 2010; 224(4): 230-235.

43 Peng X-J, Su L-P. Characteristics of fundus autofluorescence in cystoid macular edema. Chin Med J 2011; 124(2): 253-257.
44 Chung H, Park B, Shin HJ, Kim HC. Correlation of fundus autofluorescence with spectral-domain optical coherence tomography and vision in diabetic macular edema. Ophthalmology 2012; 119(5): 1056-1065.

45 Shen Y, Xu X, Liu K. Fundus autofluorescence characteristics in patients with diabetic macular edema. Chin Med J 2014; 127(8): 1423-1428.

46 Vujosevic S, Bottega E, Casciano M, Pilotto E, Convento E, Midena E. Microperimetry and fundus autofluorescence in diabetic macular edema: subthreshold micropulse diode laser versus modified early treatment diabetic retinopathy study laser photocoagulation. Retina 2010; 30(6): 908-916.

47 Vujosevic S, Casciano M, Pilotto E, Boccassini B, Varano M, Midena E. Diabetic macular edema: fundus autofluorescence and functional correlations. Invest Ophthalmol Vis Sci 2011; 52(1): 442-448.

48 Waldstein SM, Hickey D, Mahmud I, Kiire CA, Charbel IP, Chong NV. Two-wavelength fundus autofluorescence and macular pigment optical density imaging in diabetic macular oedema. Eye 2012; 26(8): 1078-1085.

49 Yoshitake S, Murakami T, Horii T, Uji A, Ogino K, Unoki N et al. Qualitative and quantitative characteristics of nearinfrared autofluorescence in diabetic macular edema. Ophthalmology 2014; 121(5): 1036-1044.

50 Ayata A, Tatlipinar S, Kar T, Unal M, Ersanli D, Bilge AH. Near-infrared and short-wavelength autofluorescence imaging in central serous chorioretinopathy. $\mathrm{Br} \mathrm{J}$ Ophthalmol 2009; 93(1): 79-82.

51 Dinc UA, Tatlipinar S, Yenerel M, Gorgun E, Ciftci F. Fundus autofluorescence in acute and chronic central serous chorioretinopathy. Clin Exp Optom 2011; 94(5): 452-457.

52 Framme C, Walter A, Gabler B, Roider J, Sachs HG, Gabel VP. Fundus autofluorescence in acute and chronicrecurrent central serous chorioretinopathy. Acta Ophthalmol Scand 2005; 83(2): 161-167.

53 Imamura Y, Fujiwara T, Spaide RF. Fundus autofluorescence and visual acuity in central serous chorioretinopathy. Ophthalmology 2011; 118(4): 700-705.

54 Lindner E, Weinberger A, Kirschkamp T, El-Shabrawi Y, Barounig A. Near-infrared autofluorescence and indocyanine green angiography in central serous chorioretinopathy. Ophthalmologica 2012; 227(1): 34-38.

55 Pang CE, Shah VP, Sarraf D, Freund KB. Ultra-widefield imaging with autofluorescence and indocyanine green angiography in central serous chorioretinopathy. Am J Ophthalmol 2014; 158(2): 362-371.

56 Spaide RF, Klancnik JM Jr. Fundus autofluorescence and central serous chorioretinopathy. Ophthalmology 2005; 112(5): 825-833.

57 Teke MY, Elgin U, Nalcacioglu-Yuksekkaya P, Sen E, Ozdal P, Ozturk F. Comparison of autofluorescence and optical coherence tomography findings in acute and chronic central serous chorioretinopathy. Int J Ophthalmol 2014; 7(2): 350-354.

58 von Ruckmann A, Fitzke FW, Fan J, Halfyard A, Bird AC. Abnormalities of fundus autofluorescence in central serous retinopathy. Am J Ophthalmol 2002; 133(6): 780-786.

59 Fung A, Kumar N, Mrejen S, Marsiglia M, Loh B, Spaide R. Retinal pigment epithelial cell loss assessed by fundus autofluorescence imaging in patients with neovascular age-related macular degeneration. Clin Exp Ophthalmol 2012; 40(Suppl 1): 49. 
60 Mehta H, Davidson A, Devary S, Egan C, Hykin P, Moore A et al. Autofluorescence and spectral-domain OCT findings in an atrophic maculopathy associated with pseudoxanthoma elasticum. Clin Exp Ophthalmol 2012; 40(Suppl 1): 49-50.

61 Rofagha S, Bhisitkul RB, Boyer DS, Sadda SR, Zhang K. SEVEN-UP Study Group Seven-year outcomes in ranibizumab-treated patients in ANCHOR, MARINA, and HORIZON: a multicenter cohort study (SEVEN-UP). Ophthalmology 2013; 120 (11):2292-2299.

62 Extension study for the evaluation of finasteride in the treatment of chronic central serous chorioretinopathy (CSC-Ext). Clinical Trials gov, 2013. Available at: https:// clinicaltrials.gov/ct2/show/NCT01227993.

63 A study of lampalizumab intravitreal injections administered every two weeks or every four weeks to patients with geographic atrophy. Clinical Trials gov, 2015. Available at: https://clinicaltrials.gov/ct2/show/NCT02 288559.

64 Ozmert E, Batioglu F. Fundus autofluorescence before and after photodynamic therapy for chronic central serous chorioretinopathy. Ophthalmologica 2009; 223(4): 263-268.

65 Brito PN, Gomes NL, Vieira MP, Faria PA, Fernandes AV, Rocha-Sousa A et al. Possible role for fundus autofluorescence as a predictive factor for visual acuity recovery after epiretinal membrane surgery. Retina 2014; 34(2): 273-280.

66 Frampton G, Kalita N, Payne E, Colquitt J, Loveman E. Accuracy of fundus autofluorescence imaging for the diagnosis and monitoring of retinal conditions: a systematic review. Health Technol Assess 2016; 20(31): 1-107.

67 Southampton Health Technology Assessments Centre (SHTAC). HTA 14/151/02: the use of fundus autofluorescence imaging for retinal conditions (systematic review protocol), 2014.

68 Reitsma JB, Rutjes AWS, Whiting P, Vlassov VV, Leeflang MMG, Deeks JJ. Chapter 9. Assessing methodological quality. In: Deeks JJ, Bossuyt PM, Gatsonis C (eds). Cochrane Handbook for Systematic Reviews of Diagnostic Test Accuracy Version 1002009.

69 Whiting P, Rutjes AW, Reitsma JB, Bossuyt PM, Kleijnen J. The development of QUADAS: a tool for the quality assessment of studies of diagnostic accuracy included in systematic reviews. BMC Med Res Methodol 2003; 3: 25.

70 Cachulo L, Silva R, Fonseca P, Pires I, Carvajal-Gonzalez S, Bernardes $\mathrm{R}$ et al. Early markers of choroidal neovascularization in the fellow eye of patients with unilateral exudative age-related macular degeneration. Ophthalmologica 2011; 225(3): 144-149.

71 Hogg RE, Silva R, Staurenghi G, Murphy G, Santos AR, Rosina $C$ et al. Clinical characteristics of reticular pseudodrusen in the fellow eye of patients with unilateral neovascular age-related macular degeneration. Ophthalmology 2014; 121(9): 1748-1755.

72 Smith RT, Chan JK, Busuoic M, Sivagnanavel V, Bird AC, Chong NV. Autofluorescence characteristics of early, atrophic, and high-risk fellow eyes in age-related macular degeneration. Invest Ophthalmol Vis Sci 2006; 47(12): 5495-5504.

73 Ueda-Arakawa N, Ooto S, Tsujikawa A, Yamashiro K, Oishi A, Yoshimura N. Sensitivity and specificity of detecting reticular pseudodrusen in multimodal imaging in Japanese patients. Retina 2013; 33(3): 490-497.
74 Vujosevic S, Trento B, Bottega E, Urban F, Pilotto E, Midena E. Scanning laser ophthalmoscopy in the retromode in diabetic macular oedema. Acta Ophthalmol 2012; 90(5): e374-e380.

75 Viswanathan M, Berkman ND, Dryden DM, Hartling L. Assessing risk of bias and confounding in observational studies of interventions or exposures: further development of the RTI item bank. Methods research report. RTI-UNC Evidence-based Practice Centre: Rockville, MD, USA, 2013. Report no. 13-EHC106-EF, AHRQ.

76 Virgili G, Menchini F, Murro V, Peluso E, Rosa F, Casazza G. Optical coherence tomography (OCT) for detection of macular oedema in patients with diabetic retinopathy (review). Cochrane Database Syst Rev 2011; Issue 7. Art. No.:CD008081.

77 Akuffo KO, Beatty S, Stack J, Peto T, Leung I, Corcoran L et al. Concordance of macular pigment measurement using customized heterochromatic flicker photometry and fundus autofluorescence in age-related macular degeneration. Invest Ophthalmol Vis Sci 2015; 56(13): 8207-8214.

78 Ben Moussa N, Georges A, Capuano V, Merle B, Souied EH, Querques G. MultiColor imaging in the evaluation of geographic atrophy due to age-related macular degeneration. Br J Ophthalmol 2015; 99(6): 842-847.

79 Cukras C, Huynh N, Vitale S, Wong WT, Ferris FL 3rd, Sieving PA. Subjective and objective screening tests for hydroxychloroquine toxicity. Ophthalmology 2015; 122(2): 356-366.

80 De Bats F, Mathis T, Mauget-Faysse M, Joubert F, Denis P, Kodjikian L. Prevalence of reticular pseudodrusen in agerelated macular degeneration using multimodal imaging. Retina 2016; 36(1): 46-52.

81 Hoeg TB, Moldow B, Klein R, La Cour M, Klemp K, Erngaard $\mathrm{D}$ et al. An evaluation of fundus photography and fundus autofluorescence in the diagnosis of cuticular drusen. Br J Ophthalmol 2016; 100(3): 378-382.

82 Liu CF, Liu L, Lai CC, Chou JC, Yeh LK, Chen KJ et al. Multimodal imaging including spectral-domain optical coherence tomography and confocal near-infrared reflectance for characterization of lacquer cracks in highly myopic eyes. Eye 2014; 28(12): 1437-1445.

83 Malamos P, Masaoutis P, Georgalas I, Maselos S, Andrianopoulos K, Koutsandrea $\mathrm{C}$ et al. The role of fundus autofluorescence imaging in the study of the course of posterior uveitis disorders. Biomed Res Int 2015; 2015: 247469.

84 Ooto S, Suzuki M, Vongkulsiri S, Sato T, Spaide RF. Multimodal visual function testing in eyes with nonexudative age-related macular degeneration. Retina 2015; 35(9): 1726-1734.

85 Pilotto E, Guidolin F, Convento E, Antonini R, Stefanon FG, Parrozzani R et al. En face optical coherence tomography to detect and measure geographic atrophy. Invest Ophthalmol Vis Sci 2015; 56(13): 8120-8124.

86 Sallo FB, Leung I, Clemons TE, Peto T, Bird AC, Pauleikhoff D. Multimodal imaging in type 2 idiopathic macular telangiectasia. Retina 2015; 35(4): 742-749.

87 Sato T, Suzuki M, Ooto S, Spaide RF. Multimodal imaging findings and multimodal vision testing in neovascular agerelated macular degeneration. Retina 2015; 35(7): 1292-1302.

88 Sayegh RG, Zotter S, Roberts PK, Kandula MM, Sacu S, Kreil DP et al. Polarization-sensitive optical coherence tomography and conventional retinal imaging strategies in 
assessing foveal integrity in geographic atrophy. Invest Ophthalmol Vis Sci 2015; 56(9): 5246-5255.

89 Shin JY, Choi HJ, Lee J, Choi M, Chung B, Byeon SH. Fundus autofluorescence findings in central serous chorioretinopathy using two different confocal scanning laser ophthalmoscopes: correlation with functional and structural status. Graefes Arch Clin Exp Ophthalmol 2016; 254(8): 1537-1544.

90 Steinberg JS, Gobel AP, Fleckenstein M, Holz FG, SchmitzValckenberg S. Reticular drusen in eyes with high-risk characteristics for progression to late-stage age-related macular degeneration. Br J Ophthalmol 2015; 99(9): 1289-1294.

91 Wu Z, Ayton LN, Luu CD, Baird PN, Guymer RH. Reticular pseudodrusen in intermediate age-related macular degeneration: prevalence, detection, clinical, environmental, and genetic associations. Invest Ophthalmol Vis Sci 2016; 57(3): 1310-1316.

92 Yonekawa Y, Thomas BJ, Drenser KA, Trese MT, Capone A Jr. Familial exudative vitreoretinopathy: spectral-domain optical coherence tomography of the vitreoretinal interface, retina, and choroid. Ophthalmology 2015; 122(11): 2270-2277.

93 Yoshitake S, Murakami T, Uji A, Unoki N, Dodo Y, Horii T et al. Clinical relevance of quantified fundus autofluorescence in diabetic macular oedema. Eye 2015; 29(5): 662-669.

94 Zhang P, Wang H-Y, Zhang Z-F, Sun D-J, Zhu J-T, Li J et al. Fundus autofluorescence in central serous chorioretinopathy: association with spectral-domain optical coherence tomography and fluorescein angiography. Int $J$ Ophthalmol 2015; 8(5): 1003.

95 Bossuyt P, Davenport C, Deeks J, Hyde C, Leeflang M, Scholten R. Chapter 11. Interpreting results and drawing conclusions. In: Deeks JJ, Bossuyt PM, Gatsonis C (eds). Cochrane Handbook for Systematic Reviews of Diagnostic Test Accuracy Version 092013.

96 Macaskill P, Gatsonis C, Deeks JJ, Harbord RM, Takwoingi Y. Chapter 10. Analysing and presenting results. In: Deeks JJ, Bossuyt PM, Gatsonis C (eds). Cochrane Handbook for Systematic Reviews of Diagnostic Test Accuracy Version 102010.

97 Gerson JD. The clinical utility of fundus autofluorescence imaging. In: The 9th Annual Guide to Retinal Disease; Rev Optom online supplement April 2012: 3-5. Available at: https:/ / www.reviewofoptometry.com/CMSDocuments/ 2012/4/0412_RetinaGuide.pdf.

98 Fleckenstein M, Schmitz-Valckenberg S, Holz F. Fundus autofluorescence imaging in clinical use. Rev Ophthalmol 2010; Available at: https:/ / www.reviewofophthalmology.com/ article/fundus-autofluorescence-imaging-in-clinical-use.

99 Bossuyt PM, Irwig LM, Craig J, Glasziou P. Comparative accuracy: assessing new tests against existing diagnostic pathways. BMJ 2006; 332: 1089-1092.

100 Ilginis T, Clarke J, Patel PJ. Ophthalmic imaging. Br Med Bull 2014; 111(1): 77-88.

Supplementary Information accompanies this paper on Eye website (http://www.nature.com/eye) 